\title{
Effects of Turbulence Modelling on 3D Flow Generated by a Piezoelectric Fan
}

\author{
Myong Hun Oh, Soo Hyun Park, Minsuk Choi \\ Department of Mechanical Engineering, Myongji University \\ 116 Myongji-Ro, Cheoin-Gu, Yongin 17058, Republic of Korea \\ mchoi@mju.ac.kr
}

\section{Extended Abstract}

Along with the miniaturization of electronics, the size of a cooling fan has also been decreasing. However, the rotary fan used for cooling the heat source has a size-limit, below which the performance of the fan decreases significantly. Recently, a vibrating plate by piezoelectric materials is considered to become an alternative to a rotary fan for cooling small electronics, because a piezoelectric fan can generate sufficient airflow for cooling in spite of its small size.

Since Toda $[1,2]$ firstly showed that a piezoelectric fan can be used for cooling a transistor, many researchers have conducted experiments and numerical simulations for understanding the 3D flow around it or analysing its cooling effects on a heated surface. In particular, Choi et al. [3] and Oh et al. [4] conducted 2D and 3D simulations for the flow around a piezoelectric fan, respectively, and compared their predicted flow data to an experimental data of Kim et al. [5]. They found that a pressure difference across the fan tip plays an important role in generating a counter-rotating vortices and also showed a detailed 3D vortical structure around the piezoelectric fan. As shown in Oh et al. [4], two kinds of vortices are generated simultaneously from the tip and the side edge of the fan, and the tip vortex and the side vortex interact severely to each other. Therefore, it is very important to predict the vortical structures as accurately as possible to investigate the 3D flow around the piezoelectric fan.

In this work, the 3D flow around a piezoelectric fan has been calculated with different turbulence modelling such as k$\varepsilon$ RNG model, k- $\omega$ model, k- $\omega$ SST model and LES (Large Eddy Simulation), and the numerical results have been compared with each other and the experimental data, in order to analyse the effects of the turbulence modelling on the flow. The predicted flow by k- $\varepsilon$ RNG model and k- $\omega$ SST model with URANS(unsteady Reynolds-averaged Navier-Stokes) simulations is quite similar to the experimentally observed one qualitatively and quantitatively, while the predicted flow by $\mathrm{k}-\omega$ model is poor in comparison to the other two models. LES showed a much more detailed flow field with a small scale flow structure than URANS with three turbulence model, but it required much more time to get a converged solution. Based on the numerical results with various numerical methods, an attempt has been made to understand the 3D flow around a piezoelectric fan for cooling small electronics.

\section{Acknowledgements}

This research was supported by Basic Science Research Program through the National Research Foundation of Korea (NRF) funded by the Ministry of Education, Republic of Korea (No.2016R1D1A1B03932666).

\section{References}

[1] M. Toda, "Theory of air flow generation by a resonant type $\mathrm{PVF}_{2}$ bimorph cantilever vibrator," Ferroelectrics, vol. 22, pp. 911-918, 1978.

[2] M. Toda, "Voltage-induced large amplitude bending device - $\mathrm{PVF}_{2}$ bimorph - its properties and applications," Ferroelectrics, vol. 32, pp. 127-133, 1981.

[3] M. Choi, C. Cierpka and Y. -H. Kim, "Vortex formation by a vibrating cantilever," Journal of Fluids and Structures, vol. 31, pp. 67-78, 2012.

[4] M. H. Oh, S. H. Park, Y. -H. kim and M. Choi, "3D flow structure around a piezoelectrically oscillating flat plate," European Journal of Mechanics - B/Fluids, vol. 67, pp. 249-258, 2018. 
[5] Y. -H. Kim, S. T. Wereley and C. - H. Chun, "Phase-resolved flow field produced by a vibrating cantilever plate between two endplates," Physics of Fluids, vol. 16, pp. 145-162, 2004. 